\title{
Postural balance and neck angle changes in school children while carrying a traditional backpack versus a double-sided bag
}

\author{
Dalia Mohammed Mosaad ${ }^{1}$, Amr Almaz Abdel-aziem² \\ ${ }^{1}$ Department of Basic Science, Faculty of Physical Therapy, Cairo University, Giza, Egypt; ${ }^{2}$ Department of Biomechanics, \\ Faculty of Physical Therapy, Cairo University, Giza, Egypt
}

\section{Summary}

Study aim: This study compared the body balance and neck angle differences in children when carrying a traditional backpack versus a double-sided bag.

Material and methods: By using the Biodex balance system and a three dimensional motion analysis system, the postural balance and neck angles were assessed in 33 school children while carrying a traditional backpack or a double-sided bag, weighing $15 \%$ of body weight.

Results: The overall and anteroposterior stability indices were significantly higher when carrying a traditional backpack compared to no load and a double-sided bag $(p<0.05)$. The mediolateral stability index was significantly higher when carrying the traditional backpack and the double-sided bag compared to no load $(\mathrm{p}<0.05)$. The craniohorizontal angle was significantly greater, and the craniovertebral angle and sagittal shoulder posture were significantly lesser when carrying the traditional backpack compared to no load and the double-sided bag conditions $(p<0.05)$.

Conclusion: Carrying the double-sided bag restores the body balance and head posture to a condition that is similar to the no load condition.

Keywords: Children - Body position - Balance - Neck angles - Load carriage

\section{Introduction}

Children use a backpack from the first day they go to school [32]. The backpack makes the load symmetrically distributed around the spine, whilst maintaining stability [20]. Students carry their educational loads mostly in backpacks, without considerations of the workplace standards that have been developed for adults [14]. So, carrying heavy bags may cause musculoskeletal disorders and balance problems among students [43].

Prolonged improper backpack loading during the childhood years makes the spine susceptible to injury. So, proper backpack use should be emphasized during these years. When the backpack load is positioned posteriorly to the body, the center of gravity (COG) shifts posteriorly over the base of the support [21]. This shifting is associated with either leaning forward at the ankle or hip or inclining the head, and contraction of postural muscles to control these adjustments to support the backpack load [42].

Continuous poor postural compensations can lead to musculoskeletal imbalances and pain. Forward head posture (FHP) occurs when the head is held anterior to its neutral, balanced position and stresses the cervical vertebrae and posterior neck muscles [16]. The forward flexion of the trunk may also lead to low back pain (LBP), due to increased stress on the ligaments and intervertebral discs of the lumbar region [30].

Many studies have reported that carrying a backpack weighing $7.5 \%$ of body weight (BW) or more leads to postural compensations $[28,29,36]$. During static conditions there is a correlation between the increased weight of the backpack and increasing the FHP, trunk flexion, spinal asymmetry, and tensile forces in the intervertebral discs [4, 37]. Mastalerz et al. [24] suggested that carrying a backpack weighing $10 \%$ of BW leads to disturbance in the plantar force distribution that decreases the postural control and stability in a standing position. Similarly, during gait there are postural changes during backpack carrying, including FHP, rounded shoulders, and forward trunk lean $[2,23]$. Moreover, the postural sways increase with increasing backpack load. These postural sways were observed when normal adolescents carried a backpack loaded with $15 \%$ of BW [40]. 
Lloyd and Cooke [22] concluded that the preservation of neutral posture during load carrying can be achieved by load distribution around the body's vertical axis. Using a double pack which allows loads to be distributed between the back and the front of the trunk leads to a smaller forward lean and displacement of the center of mass than the traditional backpack which loads the back only. Alternatively, front packs, which place the load anterior to the subject, result in an upright posture through reduction of FHP and hip flexion compared to the traditional backpacks [11]. However, the front packs increase thoracic kyphosis [46].

Recently, Abaraogu et al. [1] concluded that a $15 \%$ BW loaded backpack led to a more forward position of the head on the neck. In addition, Dahl et al. [10] reported that a nontraditional backpack (with the load placed bilaterally on the subject) created a more upright posture than the traditional backpack, and the trunk was more erect and the head less hyperextended. Moreover, nontraditional backpack posture and gait were similar to the non-loading condition.

To the best of our knowledge, the effects of a nontraditional backpack on neck angles and balance have received almost no attention in scientific literature related to children. Therefore, the aim of this study was to assess the changes in body balance and neck angles when carrying a traditional school backpack and a double-sided bag (load placed bilaterally). It was hypothesized that the doublesided bag would result in preservation of body balance and normal neck posture compared to a traditional school backpack.

\section{Material and methods}

\section{Participants}

Thirty-three right-handed children (19 male and 14 female) from primary schools participated in this study. Their mean age was $9.98 \pm 1.14$ years, mean weight $32.17 \pm 4.33 \mathrm{~kg}$, mean height $135.76 \pm 5.45 \mathrm{~cm}$, and mean body mass index (BMI) $17.36 \pm 1.50 \mathrm{~kg} / \mathrm{m}^{2}$. Each child participated in three loading conditions: no load, traditional backpack, and double-sided bag style (repeated measures design). The postural balance level and neck angles were assessed in these loading conditions by using the Biodex Stability System (BSS), and a 3D motion analysis system, respectively. The children and their parents were informed about the procedures of the study. The parents signed a consent form authorizing the child's participation. The study was performed in accordance with the Declaration of Helsinki and approved by the local institutional ethics board.

All children were physically active, able to follow the researcher's instructions, and not overweight or obese, according to the standard definition for child overweight and obesity worldwide [9]. According to the definition, the cutoff point of the mean BMI for overweight and obese children between the ages of 8.5 and 12 years is $18.76-21.22 \mathrm{~kg} / \mathrm{m}^{2}$ for boys and $18.69-21.68 \mathrm{~kg} / \mathrm{m}^{2}$ in girls. In this study, the BMI of the children ranged from 15.04 to $20.19 \mathrm{~kg} / \mathrm{m}^{2}$. Children were excluded if they had any history of back or neck pain, musculoskeletal disorders or neurological diseases.

\section{Instruments}

The Biodex Stability System (Biodex, Shirley, NY, USA) was used to assess the neuromuscular performance by evaluating the ability to remain stable on an unstable platform [45]. This system consists of a movable balance platform, which provides up to $20^{\circ}$ of surface tilt in a $360^{\circ}$ range. The motion of the unstable platform is represented as deviations from the horizontal plane [overall stability index (OSI), anterior/posterior stability index (AP), medial/lateral stability index (ML)]. The platform provides eight different stability levels, which range from a completely firm surface to a very unstable surface [38]. During motion testing, the OSI indicates the foot platform displacement in degrees. The stability index is measured as the angular excursion of a patient's COG. The variance of foot platform displacement of motion in the sagittal plane is represented by the AP stability score, and motion in the frontal plane is represented by the ML stability score. The OSI score is believed to be the best indicator of the overall ability of the participant to maintain a steady position of the platform [44].

The 3D motion analysis system consists of Pro Reflex infrared cameras, and reflective markers [eight silver colored markers (QUALISYS AB, Sweden)]. The participant stood with his/her shoulders exposed, and adhesive markers were placed on the dominant side of the body. For neck angle measurement the markers were fixed on the right eye canthus (over the eye angle), the right ear tragus, the spinous process of $\mathrm{C} 7$, and laterally to the right acromion, with two reference vertical markers (parallel to the sagittal plane), and two reference horizontal markers (parallel to the frontal plane) [28].

There were two bag styles (15\% BW): a traditional backpack, and an ergonomically designed bag (doublesided bag style). The traditional backpack was a doublestrapped backpack with dimensions of $44 \times 30 \times 15 \mathrm{~cm}$ (Fig. 1). The backpack straps were adjusted according to the child's height to place it above the hips at the lower back. The double-sided bag style is composed of a twosided bag with dimensions of $30 \times 25 \times 10 \mathrm{~cm}$. It allows vertical weight loading for a balanced upright posture and features adjustable padded shoulder straps to accommodate different body heights and an adjustable front-hip buckle closure that allows children to sit and remove the load while compartments remain at hip level (Fig. 2). 


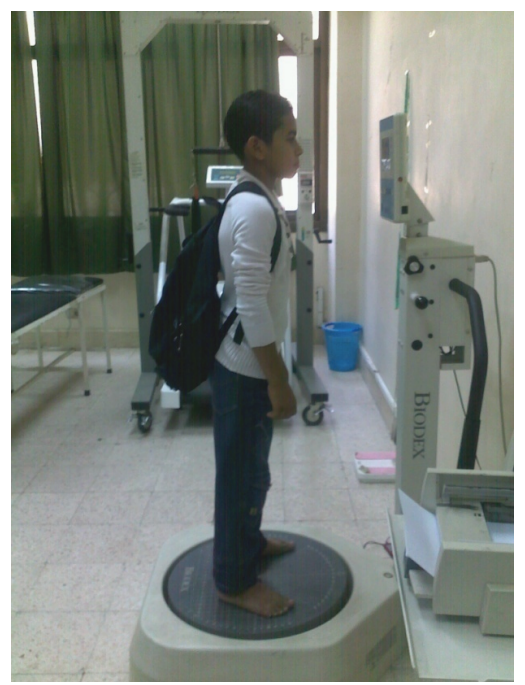

Fig. 1. Carrying a traditional backpack during balance assessment

The weight of the backpack and double-sided bag style was selected to be $15 \% \mathrm{BW}$ as most previous reports that have examined the effects of different bag weights have concluded that carrying a backpack corresponding to $15 \%$ of BW produces postural deviations [36] and carrying bags of different designs weighing $15 \%$ of BW causes adverse stress and strain on spinal structures [4]. Moreover, this load represented the average load of the previous studies $[1,4,7,10,24,26,35,36]$. An assistant prepared the bags and paid attention to ensuring a uniform vertical and horizontal load distribution to avoid load concentration and allowing the children to walk around the laboratory for five minutes, as adaptation time, to be more familiar especially with the double-sided bag style.

\section{Balance assessment}

The BSS permits the ankle joint mechanoreceptors to be stimulated maximally [17]. Platform stability levels chosen for assessment were 8 and 5 with opened eyes, as level 2 may be unstable for children. Conversely, levels 6 to 8 had superior reliability and assessment at level 5 was unstable for the participants. Therefore, the stability levels from 8 to 5 were selected [31]. The platform stability was decreased from level 8 to level 5 within 40 seconds, because lower stability levels of the device may not be appropriate for use as an objective marker of progression due to poor reliability of the scores over time [41].

The children stood barefoot on the BSS's locked platform. Then she/he was instructed to stand upright, looking straight at the system screen and assuming a comfortable erect posture with body weight evenly distributed. The child was instructed to shift the position of his/her feet until the cursor was centered on the screen grid. Then, the researcher identified the subject's foot position on the

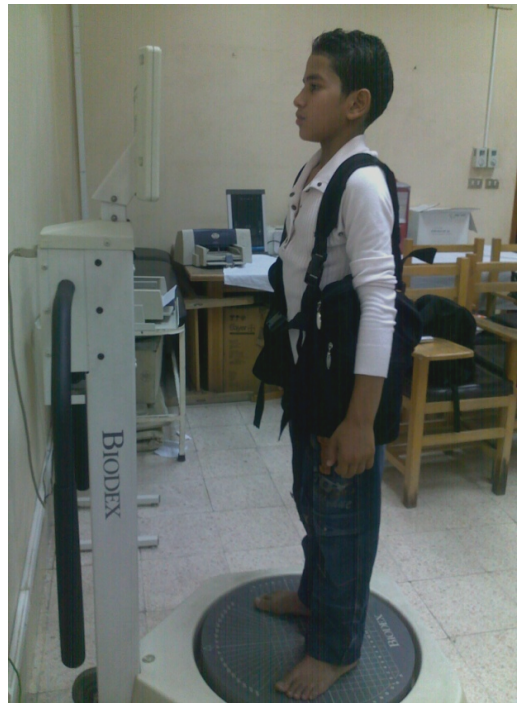

Fig. 2. Carrying the double sided bag style during balance assessment

platform through recording the heel coordinates and foot angle. The center of the heel was identified by a point of intersection of horizontal lines, represented in characters, and vertical lines, represented in numbers. The angle of the foot was determined by the line parallel to the second metatarsal. Three practice trials were completed before the first test condition. Before balance recording, the child was allowed to stand on the platform until he/she felt secure. Hence, each loading condition was tested once.

The children were instructed to rest on a chair for three minutes after each test condition to prevent fatigue that might affect the results [27]. The OSI, AP, and ML stability indices were recorded for each child under the three conditions. A high number indicated greater motion and difficulty to be stable while a low number indicates less motion and greater ability to be stable [6].

\section{Measurement of neck angles}

The neck angles during the three conditions were measured from a standing position. The children stood comfortably in the center of the walkway with bare feet and looked directly ahead, with arms beside their body. They were asked to place their weight evenly on both feet with feet $2 \mathrm{~cm}$ apart to obtain more reliable results. Then a capture of 4 seconds time was taken by the Q-trac software [28].

The craniohorizontal angle (CHA) provides an estimation of head on neck angle or position of the upper cervical spine. It is formed by the intersection of a horizontal line that passes through the tragus of the ear and a line joining the tragus of the ear and the external canthus of the eye [34]. A higher angle indicates FHP [36]. The craniovertebral angle (CVA) estimates the position of the neck on the upper trunk. A small angle indicates more FHP. It lies at the intersection of a horizontal line through the spinous 
process of $\mathrm{C} 7$ and a line to the tragus of the ear [39]. The sagittal shoulder posture (SSP) angle is composed by the intersection of a horizontal line that passes through $\mathrm{C} 7$ and a line connects the midpoint of the greater tuberosity of the humerus and the posterior aspect of the acromion. The SSP angle assesses the forward shoulder position. A smaller angle means that the shoulder lies anteriorly in relation to $\mathrm{C}$, in other words, rounded shoulder [34].

\section{Statistical analysis}

Data were analyzed using SPSS Statistics for Windows, Version 20.0 (Armonk, NY: IBM Corp). A repeated measures analysis of variance (ANOVA) was used to compare between the three loading conditions of OSI, AP, and ML stability indices and neck angles in children with no load, a traditional backpack and a double-sided bag style carriage. The least significant difference was used to determine which loading conditions mean values were significantly different from each other. The level of significance was set at $\mathrm{p}<0.05$.

\section{Results}

Descriptive statistics of the balance indices and neck angles in children with no load, a traditional backpack and a double-sided bag are presented in Table 1 and Table 2.

There was a significant difference in the $\mathrm{OSI}(\mathrm{F}=77.77$, $p=0.001), \mathrm{AP}(\mathrm{F}=63.24, p=0.001)$, and $\mathrm{ML}(\mathrm{F}=29.17$, $p=0.001)$ stability indices between the three loading conditions. Regarding the neck angles there was a significant difference in the CHA ( $\mathrm{F}=140.19, \mathrm{p}=0.001)$, CVA $(\mathrm{F}=18.87, p=0.001)$ and SSP $(\mathrm{F}=23.08, p=0.001)$ between the three loading conditions.
Post hoc analysis revealed that the mean value of OSI and AP stability indices were significantly higher when carrying the traditional backpack compared to no load and the double-sided bag $(p<0.05)$, and no significant difference between no load and the double-sided bag $(p>0.05)$. While, the mean value of the ML stability index was significantly higher when carrying the traditional backpack and the double-sided bag compared to no load $(p<0.05)$, and no significant difference between the traditional backpack and double-sided bag $(p=0.097)$.

The mean value of CHA was significantly greater when carrying the traditional backpack compared to no load and the double-sided bag $(p<0.05)$, and no significant difference between no load and the double-sided bag $(p=0.110)$. However, the mean values of CVA and SSP angle were significantly lesser when carrying the traditional backpack compared to no load and the double-sided bag $(p=0.001)$, and no significant difference between no load and the double-sided bag $(\mathrm{p}>0.05)$.

\section{Discussion}

This study was conducted to compare the effect of carrying a traditional backpack and the double-sided bag style on body balance and neck angles. The hypothesis that the double-sided bag would result in preservation of body balance and normal neck posture compared to a traditional backpack was confirmed with lower OSI and AP stability indices values when carrying the double-sided bag style than when carrying a traditional backpack. Moreover, carrying the double-sided bag style reduces CHA and increases the CVA and SSP angle, which means less FHP.

Table 1. Balance indices during different loading conditions

\begin{tabular}{lccc}
\hline Stability indices & No load & Traditional backpack & Double sided bag \\
\hline Overall stability index & $1.53 \pm 0.35$ & $1.78 \pm 0.41$ & $1.57 \pm 0.40^{*}$ \\
Anteroposterior stability index & $1.38 \pm 0.40$ & $1.62 \pm 0.39$ & $1.42 \pm 0.38^{*}$ \\
Mediolateral stability index & $1.02 \pm 0.24$ & $1.12 \pm 0.26$ & $1.10 \pm 0.25^{*}$ \\
\hline
\end{tabular}

Data are presented as mean \pm standard deviation; ${ }^{*}-\mathrm{p}<0.05$.

Table 2. Neck angles (degree) during different loading conditions

\begin{tabular}{lccc}
\hline Neck angles & No load & Traditional backpack & Double sided bag \\
\hline Craniohorizontal angle & $24.82 \pm 4.81$ & $28.70 \pm 4.64$ & $25.18 \pm 5.03^{*}$ \\
Craniovertebral angle & $45.91 \pm 5.32$ & $43.12 \pm 3.69$ & $46.39 \pm 5.13^{*}$ \\
Sagittal shoulder posture & $23.83 \pm 4.07$ & $21.65 \pm 2.93$ & $24.26 \pm 4.57^{*}$ \\
\hline
\end{tabular}

Data are presented as mean \pm standard deviation; ${ }^{*}-\mathrm{p}<0.05$. 
These results were supported by the findings of Rugelj and Sevek [39], who found that the participants' ability to maintain a steady position while standing was altered by the external load carried in the backpack. The sway linearly increased for all of the measured parameters such as sway area and medio-lateral and antero-posterior path length of the center of pressure. Increasing the backpack weight will increase the postural sway, while there is no postural sway when carrying a weight in the waist jacket. So, the carried load position is very important for controlling the postural sway.

In addition, the current results are consistent with the findings of Bakhshi [3], who evaluated the effect of backpack weight on static and dynamic balance among male students of 10- to 12-year-olds. His results indicated that there was a reduction in static and dynamic balance as well as some of the dynamic balance dimensions by using a backpack weighing $10.5 \%$ and $13 \%$ of the students' BW. Moreover, carrying backpack weights of $10 \%$ and $15 \%$ of BW led to postural sways that increased with increasing backpack load. These postural sways were observed when normal adolescents carried a backpack weighing $15 \%$ of BW. Furthermore, carrying the symmetrical backpack induced better balance compared with the asymmetric one [10].

The decreased balance when carrying a traditional backpack is consistent with the findings of Chow et al. [8], who reported that increasing the backpack load appears to affect balance predominantly in the AP direction of normal subjects. In addition, Pau et al. [32] concluded that when the applied load exceeds a certain threshold (approximately $2-4 \mathrm{~kg}$ ), that is less than the weight of backpack used in the current study, the center of pressure shifts further towards the forefoot. This is probably the result of large postural sway that enhances the unbalanced feeling originated by the presence of the backpack and unconsciously stimulates the body to overreact so as to prevent falling backwards.

The higher values of the OSI and the AP stability index when carrying a traditional backpack than when carrying the double-sided bag can be explained by the findings of Ramadan and Al-Shayea [35], who designed a new form of modified backpack. The modified double-sided school backpack has the ability to distribute carried loads by dividing the backpack into two parts. The first part is placed on the student's chest and the second part is placed on the student's back. The double-sided bag was superior to a traditional backpack in terms of less muscular activity, lower cardiac costs and lower exertion ratings.

In the same context, Heller et al. [15] assessed the changes in postural sway in females as a consequence of wearing a military backpack. There were two conditions: unloaded and while wearing an $18.1 \mathrm{~kg}$ backpack. They found an increase in the ML excursion, which is consistent with the findings of the current study, and an increase in the AP excursion, which is supported by the current results. Gillette et al. [12] reported that carrying loads bilaterally decreases the knee and hip adduction moments with increasing loads, which is not supported by the higher values of the ML stability index when carrying the traditional and the double-sided bag compared to no load condition.

The results also revealed that carrying the doublesided bag reduces the CHA value and increases the CVA and SSP angle values compared to carrying a traditional backpack, which resembles the no load condition. These results proved that there was improvement in the neck posture when carrying the double-sided bag compared to a traditional backpack. Bobet and Norman [5] reported that the addition of a pack load on the back resulted in shifting of body COG of the body backward and creating extension moments, which were counterbalanced by both a forward trunk lean and a forward head shift [13]. These findings explain the greater increase in FHP when carrying a traditional backpack compared to the double-sided bag style. The higher value of CHA when carrying a backpack concurs with the findings of Ramprasad et al. [36], who concluded that the CHA increased significantly after carrying a backpack corresponding to $10 \% \mathrm{BW}$, which was lighter than that used in the current study.

The double-sided bag placed the load in line with the vertical axis of the body, allowing the child to maintain a more upright posture. This posture permits a more natural spine curvature and thus may help to reduce the likelihood of LBP caused by flattening of the lumbar spine with trunk flexion [30]. In this study, head angles acted as indicators of the trunk angle as the trunk flexed and the head hyperextended, allowing participants to look straight ahead and not at the ground. Consequently, with backpack carrying there was a more forward trunk lean, and the head position was more hyperextended than when carrying the double-sided bag. A hyperextended neck may result in shoulder and neck pain [15]. The hyperextension position also places undue stress on the cervical vertebrae by removing the natural shock absorbing curve and directs the weight of the head straight towards the discs and posterior facet joints [16].

This result is supported by the findings of Kim et al. [18], who reported that when carrying the modified double pack, the forward head angle and forward head distance decreased when compared to carrying a traditional backpack. These findings indicate that the modified double pack minimizes the postural deviation. Carrying the double-sided bag leads to a decrease in CHA compared to carrying the same weight in the traditional backpack. This result is in line with the findings that even when carrying a light weight traditional backpack, $7.5 \%$ of BW, the CHA increased, but the increase was statistically insignificant compared to the unloaded condition [28]. 
Moreover, the current study reported that the CVA was significantly lesser when carrying a traditional backpack compared to the double-sided bag style, which is supported by the findings of Chansirinukor et al. [7], who found a significant reduction in the CVA (or increased FHP) whilst carrying a backpack weighing $15 \%$ of $\mathrm{BW}$ over both shoulders. Also, it is confirmed by the recent findings of Abaraogu et al. [1], who stated that the decrease in CVA between the no load condition and 15\% BW load was significant. Moreover, a $15 \%$ BW backpack led to a more forward posture of the head on the neck.

Carrying a traditional backpack significantly decreases the SSP angle compared to the double-sided bag. Raine and Twomey [33] mentioned that the smaller SSP angle indicated a relatively forward shoulder in relation to $\mathrm{C} 7$ and so represented a more rounded posture. Moreover, Mo et al. [26] reported that boys carrying backpacks weighing $10 \%$ and $15 \%$ of their BWs were more likely to have a FHP, rounded shoulder posture and increased lateral tilting of the shoulders during gait as backpack loads were increased. However, this finding is not supported by the results of Abaraogu et al. [1], who did not find changes in the SSP angle with backpack weight within 15\% BW. These contradictory findings can be explained by the difference in study group characteristics, their participants being young adults aged $18-25$ years.

Although the subjects who participated in the study by Dahl et al. [10] were young male adults, their findings support the nonsignificant difference between the unloaded condition and the double-sided bag of the current study. They concluded that the nontraditional backpack (backTpack) allowed the subject to maintain a more upright posture than a traditional backpack, the trunk was more erect and the head was less hyperextended. The nontraditional backpack facilitated a more upright stance that reduced the negative effects of poor posture such as neck and shoulder pain, LBP, and musculoskeletal asymmetries. The gait when carrying the nontraditional backpack is similar to the gait patterns of the participants during the no load condition.

Preserving the body balance and neck posture when carrying the double-sided bag style may be attributed to the distribution of the carried load on both sides of the body, which is consistent with the findings of McGill et al. [25], who concluded that carrying a load in one hand makes the spine more loaded than if the load were distributed between both hands. This effect is exaggerated as the magnitude of the carried load increases. Even if the subject carries twice the weight, the load on the spine will not increase if carrying the weight in both hands. They recommended balancing the load between both hands when carrying material, which appears to have advantages and should be considered when planning work.

There are some limitations that should be considered before generalization of the results of this study. The first is the small number of the school children recruited. So, a further study with a larger group will increase the generalizability of the results. Second, this study examined the immediate effects of carrying the double-sided bag and a traditional backpack on body balance and neck posture. The longer-term effects of load carriage and associated fatigue should be addressed in future studies. Finally, certain secondary outcome variables, such as cardiovascular response and energy expenditure, were not assessed in our study. Further research should focus on adolescents and recording the myoelectric activities of trunk and lower extremity muscles in order to assess the potential for the double-sided bag style to reduce the possibility of falling. However, the present study assessed the changes that occurred in the head and neck in addition to balance disturbance, so future investigations should focus on the posture of the trunk and pelvis and their relation to static and dynamic balance during walking in children while carrying a double-sided bag. More studies assessing the spine forces associated with carrying the double-sided bag should be conducted to fully explain its mechanical effects. Finally, further studies are needed to determine the effect of the double-sided bag style on gait kinematics and kinetics.

\section{Conclusion}

Carrying the double-sided bag was superior to the traditional backpack in terms of preserving the body balance, and decreasing the sagittal deviation of the head and neck posture for school children. It restores the body balance and minimizes the FHP by redistributing the carried loads so that they are similar to the no load condition. Moreover, this study provides the community with a double-sided bag that maintains the body balance and upright postural alignment that may reduce the potential negative effects of the traditional backpack, i.e. neck pain and postural deviations. So, using the double-sided bag will be more comfortable for students than the traditional backpack.

\section{Conflict of interest: Authors state no conflict of interest.}

\section{References}

1. Abaraogu U.O., Ezenwankwo E.F., Nwadilibe I.B., Nwafor G.C., Ugwuele B.O., Uzoh P.C., Ani I., Amarachineke K., Atuma C., Ewelunta O. (2017) Immediate responses to backpack carriage on postural angles in young adults: A crossover randomized self-controlled study with repeated measures. Work, 57(1): 87-93. DOI: 10.3233/ WOR-172531. 
2. Attwells R.L., Birrell S.A., Hooper R.H., Mansfield N.J. (2006) Influence of carrying heavy loads on soldiers' posture, movements and gait. Ergonomics, 49(14): 1527-1537.

3. Bakhshi M. (2016) The effect of backpack weight on static and dynamic balance among male students of 10 to 12 years old in Shahr-E Kord. Int. J. Sport Studies, 6(3): 178-182.

4. Bettany-Saltikov J., Cole L. (2012) The effect of frontpacks, shoulder bags and handheld bags on 3D back shape and posture in young university students: an ISIS2 study. Stud. Health Technol. Inform., 176: 117-121. DOI: 10.3233/978-1-61499-067-3-117.

5. Bobet J., Norman R.W. (1984) Effects of load placement on back muscle activity in load carriage. Eur. J. Appl. Physiol. Occup. Physiol., 53(1): 71-75.

6. Cachupe W.J., Shifflett B., Kahanov L., Wughalter E.H. (2001) Reliability of Biodex Balance System Measure. Meas. Phys. Educ. Exerc. Sci., 5(2): 97-108.

7. Chansirinukor W., Wilson D., Grimmer K., Dansie B. (2001) Effects of backpacks on students: Measurement of cervical and shoulder posture. Aus. J. Physiother., 47(2): 110-116.

8. Chow D.H., Kwok M.L., Cheng J.C., Lao M.L., Holmes A.D., Au-Yang A., Yao F.Y., Wong M.S. (2006) The effect of backpack weight on the standing posture and balance of schoolgirls with adolescent idiopathic scoliosis and normal controls. Gait Posture, 24(2): 173-181.

9. Cole T.J., Bellizzi M.C., Flegal, K.M., Dietz W.H. (2000) Establishing a standard definition for child overweight and obesity worldwide: international survey. B.M.J., 320(7244): 1240-1243. DOI: 10.1136/ bmj.320.7244.1240.

10. Dahl K.D., Wang H., Popp J.K., Dickin D.C. (2016) Load distribution and postural changes in young adults when wearing a traditional backpack versus the backTpack. Gait Posture, 45: 90-96.

11. Fiolkowski P., Horodyski M., Bishop M., Williams M., Stylianou L. (2006) Changes in gait kinematics and posture with the use of a front pack. Ergonomics, 49: 885-894.

12. Gillette J.C., Stevermer C.A., Miller R.H., Edwards W.B., Schwab C.V. (2012) Lower extremity joint moments during carrying tasks in children. J. Appl. Biomech., 28(2): 156-164.

13. Goh J.H., Thambyah A., Bose K. (1998) Effects of varying backpack loads on peak forces in the lumbosacral spine during walking. Clin. Biomech., 13: S26-S31.

14. Grimmer K., Dansie B., Milanse S., Ubon P., Patricia T. (2002) Adolescent standing postural response to backpack loads: A randomized controlled experimental study. BMC Musculoskelet. Disord., 3: 10. DOI: 10.1186/14712474-3-10.
15. Heller M.F., Challis J.H., Sharkey N.A. (2009) Changes in postural sway as a consequence of wearing a military backpack. Gait Posture, 30(1): 115-117.

16. Kapandji I.A. (2007) The physiology of the joints. 6th ed., English ed. Edinburgh; New York: Churchill Livingstone.

17. Karimi N., Ebrahimi I., Kahrizi S., Torkaman G. (2008) Evaluation of postural balance using the BIODEX Balance System in subjects with and without low back pain. Pak. J. Med. Sci., Part II, 24(3): 372-377.

18. Kim M.H., Yi C.H., Kwon O.Y., Cho S.H., Yoo W.G. (2008) Changes in neck muscle electromyography and forward head posture of children when carrying schoolbags. Ergon., 51(6): 890-901.

19. Kistner F., Fiebert I., Roach K., Moore J. (2013) Postural compensations and subjective complaints due to backpack loads and wear time in schoolchildren. Pediatr. Phys. Ther., 25: 15-24. DOI: 10.1097/PEP.0b013e31827ab2f7.

20. Knapik J., Harman E., Reynolds K. (1996) Load carriage using packs. A review of physiological, biomechanical and medical aspects. Appl. Ergon., 27: 207-216.

21. Lanes T., Gauron E., Spratt K., Wernimont T., Found E., Weinstein, J. (1995) Long term follow up of patients with low back pain treated in a multidisciplinary rehabilitation program. Spine, 20: 801-806.

22. Lloyd R., Cooke C. (2011) Biomechanical differences associated with two different load carriage systems and their relationship to economy. Hum. Move., 12(1): 65-74.

23. Majumdar D., Pal M.S., Majumdar D. (2010) Effects of military load carriage on kinematics of gait. Ergonomics, 53: 782-791.

24. Mastalerz A., Niźnikowski T., Buszta M.,Różański P., Wiśniowski P., Sadowski J. (2016) Effect of two backpack designs on cop displacement and plantar force distribution in children during upright stance. Pol. J. Sport Tourism, 23(3): 123-126.

25. McGill S.M., Marshall L., Andersen, J. (2013) Low back loads while walking and carrying: comparing the load carried in one hand or in both hands. Ergon., 56(2): 293-302. DOI: 10.1080/00140139.2012.752528.

26. Mo S.W., Xu D.Q., Li J.X., Liu M. (2013) Effect of backpack load on the head, cervical spine and shoulder postures in children during gait termination. Ergon., 56(12): 1908-1916. DOI: 10.1080/00140139.2013.851281.

27. Moezy A., Olyaei G., Hadian M., Razi M., Faghihzadeh S. (2008) A comparative study of whole body vibration training and conventional training on knee proprioception and postural stability after anterior cruciate ligament reconstruction. Br. J. Sports Med., 42(5): 373-378.

28. Mosaad D.M., Abdel-aziem A.A. (2015) Backpack carriage effect on head posture and ground reaction forces in school children. Work, 52: 203-209. DOI: 10.3233/ WOR-152043. 
29. Negrini S., Carabalona R. (2002) Backpacks on! Schoolchildren's perceptions of load, associations with back pain and factors determining the load. Spine, 27(2): 187-195.

30. Novak J.S. (1999) Posture, get it straight! 1st ed. New York: Perigee Books.

31. Patreno M.V., Myer G.D., Ford K.R., Hewett, T.E. (2004) Neuromuscular training improves single-limb stability in young female atheletes. J. Orthop. Sports Phys. Ther., 34: 305-316.

32. Pau M., Corona F., Leban B., Pau M. (2011) Effects of backpack carriage on foot-ground relationship in children during upright stance. Gait Posture, 33: 195-199.

33. Raine S., Twomey T.L. (1997) Head and shoulder posture variations in 160 asymptomatic women and men. Arch. Phys. Med. Rehabil., 78(11): 1215-1223.

34. Raine S., Twomey L. (1994) Posture of the head, shoulder and thoracic spine in comfortable erect standing. Aus. J. Physiother., 40: 25-32.

35. Ramadan M.Z., Al-Shayea A.M. (2013) A modified backpack design for male school children. Inter. J. Ind. Ergon., 43: 462-471. DOI: 10.1016/j.ergon.2013.03.002.

36. Ramprasad M., Alias J., Raghuveer A.K. (2010) Effect of backpack weight on postural angles in preadolescent children. Indian Pediatr., 47(7): 575-580.

37. Rodríguez-Soto A.E., Jaworski R., Jensen A., Niederberger B., Hargens A.R., Frank, L.R., Kelly K.R., Ward S.R. (2013) Effect of load carriage on lumbar spine kinematics. Spine, 38: E783-91. DOI: 10.1097/ BRS.0b013e3182913e9f.

38. Rozzi S.L., Lephart S.M., Sterner R., Kuligowski L. (1999) Balance training for persons with functionally unstable ankles. J. Orthop. Sports Phys. Ther., 29(8): 478-486.

39. Rugel J.D., Sevek F. (2008) The influence of load placement on postural sway parameters. 13th International Conference on Biomedical Engineering. Vol. 23 of the series IFMBE Proceedings, 2008, 3-6 December, Singapore pp. 1821-1824.
40. Sahli S., Rebai H., Ghroubi S., Yahia A., Guermazi M., Elleuch M.H. (2013) The effects of backpack load and carrying method on the balance of adolescent idiopathic scoliosis subjects. Spine J., 13(12): 1835-1842. DOI: 10.1016/j.spinee.2013.06.023.

41. Schmitz R., Arnold B. (1998) Intertester and interatester reliability of a dynamic balance protocol using the Biodex Stability System. J. Sport Rehabil., 7: 95-101.

42. Shumway C.A. (2001) Control of normal mobility. In: Shumway C.A., Woollacott M.H., (Eds.) Motor Control: Theory and Practical Applications. Maryland USA: Lippincott Williams and Wilkins, p. 128-234.

43. Singh T., Koh M. (2009) Effects of backpack load position on spatiotemporal parameters and trunk forward lean. Gait Posture, 29: 49-53.

44. Testerman C., Vander Griend R. (1999) Evaluation of ankle instability using the Biodex Stability System. Foot Ankle Int., 20: 317-321.

45. Vathrakokilis K., Malliou P., Gioftsidou A., Beneka A., Godolias G. (2008) Effects of a balance training protocol on knee joint proprioception after anterior cruciate ligament reconstruction. J. Back Musculoskelet. Rehabil., 21: 233-237.

46. Wang C.X.G., Chow D.H.K., Pope M.H. (2007) Biomechanical effect of load carriage on spine curvature and repositioning ability in adolescents. Anaheim, CA: ACTA Press, p. 161-166.

\section{Received 14.12.2017 \\ Accepted 10.04.2018}

(C) University of Physical Education, Warsaw, Poland

\section{Acknowledgments}

The authors wish to acknowledge their gratitude to the children, and their parents. 